\title{
Modeling and Simulation of Combined Discrete Event-Continuous Systems Using DEVS Formalism and Object-Oriented Paradigm
}

\author{
M.TEGGAR and R. SOENEN \\ G.I.L -LAMIH UVHC \\ BP 311, Le Mont Houy ,59326 - Valenciennes Cedex \\ Tél.: 27.14.13.47,E-mail: teggar@univ-valenciennes.fr
}

\begin{abstract}
This paper presents an approach to a simulation-based design methodology and is motivated by the need to provide models representation schemes and simulation software tools for combined discrete event-continuous systems. Our approach is based on an extension of the DEVS (Discrete EVent System Specification) introduced by Zeigler, to include a combined discrete event-continuous systems. We show how the interactions between the discrete-event part and the continuous part and their consequence on the system behavior can be expressed. By employing the Object-Oriented paradigm, the extended model is implemented as an abstract class, in which the methods describe the model's behavior and data represent its variables.
\end{abstract}

\section{Keywords}

Modeling and simulation, combined simulation, discrete event simulation, continuous system simulation

\section{Introduction}

In addition to discrete event models and continuous models, combined discrete-eventcontinuous modeling and simulation is finding ever more application in the analysis and design of complex manufacturing systems. A lot of complex systems, such as steel-making, metallurgy, chemical industry ..., involve both processes which are easily described by differential equations and processes which are better described by models whose changes in state occur at discrete instants rather than continuously in time.

Classical approaches to simulation typically use: 
- differential equations based models and frequency models for continuous systems (continuous states, continuous time),

- state-transition, automaton and Markovian models for discrete-time and discrete-events dynamic systems.

Unfortunately, these different models are incompatible because they deal with variables which don't belong to the same mathematical space, and the time variable is not used in an identical manner.

In fact, the same physical system may be modeled in an entirely continuous or an entirely discrete fashion depending on the modeler point of view and the duration for which the system will be observed. However, many works, (Wang, 89),(Cellier, 79), (Fahrland, 70), show that there exist classes of problems which can not be modeled satisfactorily by either a purely discrete or a purely continuous formulation. And the choice between the two points of view is not always easy, since any system optimization study must be global and the continous part and the discrete part of a process are often closely linked (Caristi, 91).

Although many formalisms have been developed to study, on the one hand discrete event dynamic systems and on the other hand, continuous system, there is no formulation that meets them all. Indeed, each of these various formalisms can be viewed as offering a potential application benefit based on the particular form of abstraction implicit in its "world of view". However, since reality does not usually constrain itself to one such "world view", the need to formulate representation schemes which allow to bridge the gap between the two description form becomes essential.

Two main approaches to the modeling of combined systems have recently emerged :

- defining a single formalism which encompasses the discrete event and continuous behavior and which uses a homogeneous model structure (Praehofer, 90, 92) (Zeigler, 89), (Buisson, 93)

- using a specific formalisms for each class of system components and defining an appropriate interface between them (Stiver, 93) (Antsaklis, 93) (Alla, 94).

The DEVS formalism, initially developed for formalizing simulation models of discrete event systems to be simulated, supports an open approach for the exploration of new simulationbased representations for many classes of systems. It provides a mechanism for the specification of simulation models in a modular and hierarchical manner and represents an alternative to the traditional worldviews of discrete-event-simulation languages.

To shed some light on the DEVS formalism (Zeigler, 76), a conceptual framework underlying the formalism is first described. We illustrate how basic models are specified and how these models are connected together in hierarchical fashion to form complex models. Then we show how continuous states are introduced to extend the DEVS expressibility to combined discretecontinuous systems. Thus, the extended scheme represents both types of system and the interactions between them in the same modeling framework.

After developing a mapping of the extended scheme onto a generic description so that classes of objects form a toxonomical hierarchy in which they are arranged according to their degree of generality, we describe an implementation of the simulation strategy using the abstract simulators principles (Concepcion, 88) applied to discrete event-continuous simulation. 


\section{THE DEVS FORMALISM}

DEVS (Discrete EVent System Specification) (Zeigler, 76, 84, 87) is a set-theoretic based formalism that provides a system theoretically grounded means of expressing hierarchical and modular discrete event simulation. It is the shorthand formulation needed to specify systems whose input, state and output trajectories are piecewise constant.

\subsection{Basic model}

In this formalism a basic model (called atomic model) is defined by the structure:

where

$$
M=<X, S, Y, \delta_{i n t}, \delta_{\text {ext }}, \lambda, t a>\text {, }
$$

$X=$ set of external input event

$S=$ set of sequential states

$Y=$ set of output

$\delta_{i n t}: S \rightarrow S$ is the internal transition function

$\delta_{\text {ext }}: Q \times X \rightarrow S$ is the external transition function

where $Q=\{(s, e) \mid s \in S, 0 \leq e \leq t a(s)\}$ is the total state set

$\lambda: S \rightarrow Y$ is the output function

ta: $S \rightarrow R_{Q}^{+}$the time advance function.

There are two kinds of events; external events (input events) and internal events which are time scheduled events. For each state $s$ the time advance function $t a$, which is a mapping from state space $S$ to positive real numbers, defines the time interval to the next internal event. When this time given by $t a(s)$ has elapsed, an internal event occurs. The system produces an output $\lambda(s)$ and the internal transition function specifies the next state $s^{\prime}=\delta_{\text {int }}(s)$ to which the system will transit. If an external event (an input) occurs at elapsed time $e$ which is less than $t a(s)$ time units, then a new state $s^{\prime}$ is computed by means of the external transition function $\delta_{e x t}(s, e, x)$.

\subsection{Multicomponent Model}

Several atomic models can be coupled to form a multicomponent model defined by the structure:

$D N=<D,\left\{M_{\alpha}\right\},\left\{I_{\alpha}\right\} ;\left\{Z_{\alpha, \beta}\right\}$, select $>$

where

$D=$ set of component names,

For each $\alpha$ in $D$

$M_{\alpha}=$ the component model

$I_{\alpha} \subseteq D$ set of influences of $\alpha$

and for each $\beta$ in $I_{\alpha}$

$Z_{\alpha, \beta}$ is the $\alpha$-to- $\beta$ output translation function

select : $2^{D} \rightarrow D$ is the tiebreaking selector function. It selects a component from the imminent components having the minimum next event time. This component is then allowed to execute its next event transition.

A coupled model $D N$ can be expressed as an equivalent atomic model and can itself be employed in a larger coupled model. This shows that the DEVS formalism is closed under 
coupling and hence the construction of modular hierarchical models is possible and formally well defined.

\section{EXTENDED DEVS EXPRESSIBILITY TO CONTINUOUS STATES SYSTEMS}

In this section, we introduce an extension of DEVS formalism to combined systems whose input and output are eventlike. The systems contain both discrete states and continuous states and the dynamic behaviour of the continuous components can be described by differential equations.

\subsection{Example : The Bottle-filling process}

Consider a cylindrical tank with two threshold sensors at two levels called $L$ (Low) and $H$ (High) and a bottle conveyer as it shown in the figure 1 below;

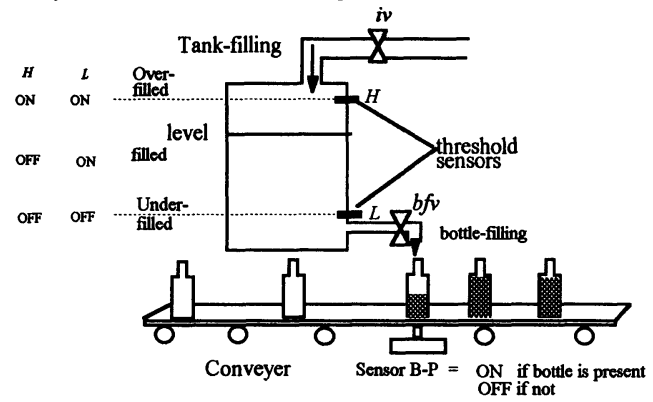

Figure 1. Bottle -filling example.

The filling process receives three types of event:

$\mathrm{e}_{11} / \mathrm{e}_{12}$ : Open_influx_valve/Close_influx_valve ;

$e_{21} / e_{22}$ : Stop_on_error/ Re-Start; (from the higher level Control System.)

$e_{3}$ : Bottle_Arrived ; (from the conveyer when an empty bottle arrive under the tank's outlet.)

And it transmits

- an event $o_{1}=$ move_conveyer when a bottle is full ( to the conveyer)

- and two events $o_{2}=$ Underfilled and $o_{3}=$ Overfilled (to the higher level control system.)

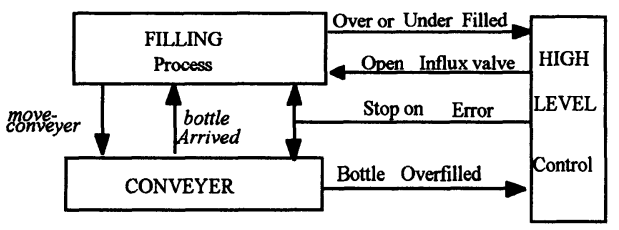

Figure 2. A coupled discrete event-continuous system. 
When the filling process receives an event $e_{11}$ the influx valve (iv) is fully open and the tank is filled with constant flow $F_{i n}$. $i v$ is closed when $e_{12}$ is received. When it receives an event $e_{22}$; this means that there is an error (bottle overflow, a failure of sensors, a leak in the tank,...) then the filling process is stopped. Event $e_{3}$ indicates a bottle-arrival, then the bottle-filling-valve (bfv) is fully open; the flow out is given by $F_{\text {out }}(t)=R . l(t)(R$ is the fill rate, some positive real number and $l(t)$ is the level of the liquid in the tank). When the volume of the bottle is reached the $b f v$ is closed and an event move_conveyer is sent to the conveyer.

\section{$3.2 \quad$ Extended DEVS Model}

The example shows that, in addition to the sequential states, the total state contains some continuous variables (Figure 3) (the level of the liquid in the tank and the volume out in the filling process).

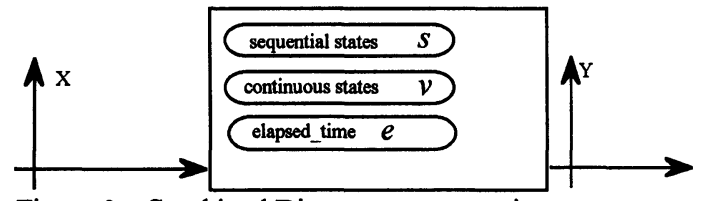

Figure 3 - Combined Discrete event-continuous system.

Moreover, two types of internal event are distinguished :

- The state-events, which are not time scheduled events but are caused by the continuous changes of the continuous states.

- The time events, which are time scheduled as in the DEVS definition given above.

A Combined Dynamic System is a tuple $\langle X, Q, Y, E, \delta, \mathcal{F}, \lambda, \sigma, t a\rangle$ where :

- $X$ is a finite set of input events and $Y$ is a finite set of output events

- $E$ is a finite set of internal state-events;

- $Q$ is the state set range defined by : $Q=S \times R^{n} \times R^{+}, S$ is discrete state set and $R^{\mathrm{n}}$ is a continuous state-space;

$$
Q=\left\{q=(s, v, e) \mid s \in S, v \in R^{n}, e \in R_{\infty}^{+}\right\}
$$

- The conditions of occurrence of internal state-events are given by the function $\sigma$ which associates at each $e_{s} \in E$ a predicate $P(q)$ where $q$ is the total state variable and $P(q)$ is a logical expression

Whenever the condition $P(q)$ becomes true, an internal state-event occurs.

- An internal time event $\varepsilon$ occurs if the condition $e \geq t a(s)$ becomes true

- $\delta$ is the discrete state transition defined by the two functions $\delta_{\text {int }}$ and $\delta_{\text {ext }}$ as follows :

$$
\begin{aligned}
& \delta_{\text {ext }}: Q \times X \rightarrow Q ; \\
& \delta_{\text {int }}: S \times E \bigcup\{\varepsilon\} \rightarrow Q ;
\end{aligned}
$$

- $\mathcal{F}$ is the differential equation defining the rate of change of the continuous state variables;

- $\lambda: \mathrm{S} \rightarrow \mathrm{Y}$ is the output function 


\subsection{The Filling-Process Model}

For the filling process described above, the sets $X, Y$ are defined by :

$\begin{aligned} X & =\left\{e_{11}, e_{12}, e_{21}, e_{22}, e_{3}\right\} \\ Y=\left\{o_{1}, o_{2}, o_{3}\right\} & {\left[\begin{array}{c}L \\ H \\ b f v \\ i v \\ \text { phase }\end{array}\right] \text { denote the sequential state and } v=\left[\begin{array}{c}l \\ v_{-} \text {out }\end{array}\right] \text { denote the continuous state } }\end{aligned}$

where $: L, H$, phase $\in\{O N, O F F$; phase $=O F F$ if the system is stopped ; $O N$ otherwise; bfv, iv $\in\{0,1\}$, the two variables take the value 0 when the corresponding valve is closed and 1 if it is fully open

the total state set is

$$
Q=\left\{q=\left(s^{T}, v^{T}, e\right)\right\}=S \times R^{2} \times R_{\infty}^{+} ; S=\{O N, O F F\} \times\{O N, O F F\} \times\{0,1\} \times\{0,1\} \times\{O N, O F F\} ;
$$

The set $E$ of the state-events contains five events : $E=\left\{e_{S_{1}}, e_{S_{2}}, e_{S_{3}}, e_{S_{4}}, e_{S_{5}}\right\}$ and $\sigma$ is given by :

$$
\begin{aligned}
& e_{s_{1}} \mapsto l \geq H I G H \\
& e_{s_{2}} \mapsto l<H I G H \\
& e_{s_{3}} \mapsto l>L O W \\
& e_{s_{4}} \mapsto l \leq L O W \\
& e_{s_{s}} \mapsto v_{-} \text {out } \geq B_{-} \text {volume } \\
& \operatorname{ta}(s)=\infty, \forall s \in S \text { No time event }
\end{aligned}
$$

The external transition function $\delta_{\text {ext }}$ is :

$\left((L, H, b f v, 0, O N), v, e, e_{11}\right) \mapsto((L, H, b f v, l, O N), v, 0)$

$\left((L, H, b f v, l, O N), v, e, e_{12}\right) \mapsto((L, H, b f v, 0, O N), v, 0)$

$\left((L, H, b f v, i v, O N), v, e, e_{2 l}\right) \mapsto((L, H, 0,0, O F F), v, 0)$

$\left((L, H, b f v, i v, O F F), v, e, e_{22}\right) \mapsto((L, H, 0,0, O N), v, 0)$

$\left((L, H, 0, i v, O N), v, e, e_{3}\right) \mapsto((L, H, 1, i v, O N), v, 0)$

the internal transition function is defined by :

$$
\begin{aligned}
& \left((L, O F F, b f v, i v, O N), e_{s_{1}}\right) \mapsto((L, O N, b f v, i v, O N), v, 0) \\
& \left((L, O N, b f v, i v, O N), e_{s_{2}}\right) \mapsto((L, O F F, b f v, i v, O N), v, 0) \\
& \left((O F F, H, b f v, i v, O N), e_{s_{3}}\right) \mapsto((O N, H, b f v, i v, O N), v, 0) \\
& \left((O N, H, b f v, i v, O F F), e_{s_{4}}\right) \mapsto((O F F, H, b f v, i v, O N), v, 0) \\
& \left((L, H, 1, i v, O N), e_{s_{s}}\right) \mapsto((L, H, 0, i v, O N),(l, 0), 0)
\end{aligned}
$$

The differential equations are given by :

$$
\mathcal{F} \equiv\left\{\begin{array}{l}
\frac{d l}{d t}=\frac{I}{T S} \cdot\left[F_{\text {in }} \cdot i_{-} v-R \cdot l(t) \cdot b_{-} f_{-} v\right] ; \text {; } \text { of the tank } \\
\frac{d v_{-} \text {out }}{d t}=R \cdot l(t) \cdot b_{-} f_{-} v
\end{array}\right.
$$


the output function :

$\lambda(O F F, O F F, b f v, i v, O N)=\left(o_{2}, H_{-} L_{-} C\right) ;$ Tank underfilled (to Higher level control)

$\lambda(O N, O N, b f v, i v, O N)=\left(o_{3}, \mathrm{H}_{-} \mathrm{L}_{-} \mathrm{C}\right) ; \quad$ Tank_overfilled (to Higher level control)

$\lambda(\mathrm{L}, \mathrm{H}, 0, i v, O N)=\left(o_{1}\right.$, Conveyer); move_conveyer (to CONVEYER)

$\lambda(\mathrm{s})=\phi ;$ for the other cases $(\phi=$ no event)

\section{SIMULATION WORLD VIEW}

The Object-Oriented Concept is not only a useful programming style and an efficient way to organize software systems, but it is also regarded as a powerful paradigm that can serve as a useful way to express computational models for large, complexly interacting systems. Encapsulation, inheritance, abstraction and evolution of individual objects support concurrency, incremental modifiability and reusability of models. Thus, it provides a common basis that can be used to implement modular simulation programs.

The simulation strategy for DEVS models is based on the abstract simulator principles developed by Zeigler et al (Zeigler, 84) (Concepcion, 85, 88) (Kim, 88, 89). The abstract simulator includes the algorithmic interpretation of the dynamic behaviour implicitly specified by the model.

The abstract simulator has the same structure as the hierarchical structure of the DEVS models. Thus the model can be directly transformed into an executable simulation program using the object oriented concept. A full explication and the class specialization hierarchy are given in (Zeigler, 90).

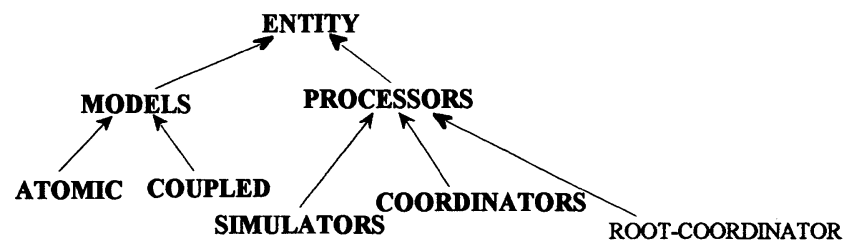

Figure 4. The abstract classes hierarchy.

The root class ENTTTY provides general definitions for data and methods of general utility. The class MODELS which in turn is specialized into more specific classes ATOMIC and COUPLED provide the basic constructs needed to specify a model's variables (Instance variables) and its behavior (methods). The class PROCESSORS and its subclasses implement methods to carry out the simulation and compute the states and outputs trajectories.

Using the same methodology, we have developed an implementation using the $\mathrm{C}++$ language according to the class hierarchy shown in figure 4 (Teggar, 96). Since these classes are defined as abstract classes, they are implemented in generic form as template classes. The methods expressing a specific behavior of user model are defined as virtual functions with template data types. 


\subsection{The class combined model}

The inheritance mechanism supports incremental specification and composition of behaviour so that user methods are defined as subclasses, which inherit the code representing data and behaviour common to the other models. The class for Combined models is a specialization of the class ATOMIC in which we can add new instance variables for the continuous states and new methods :

- sigma : which returns a state event identifier when the associated condition becomes true and 0 otherwise - derivative : computes the derivative values of the continuous states .

Using pseudo-code, we describe the class of the filling process model as a subclass of combined_models.

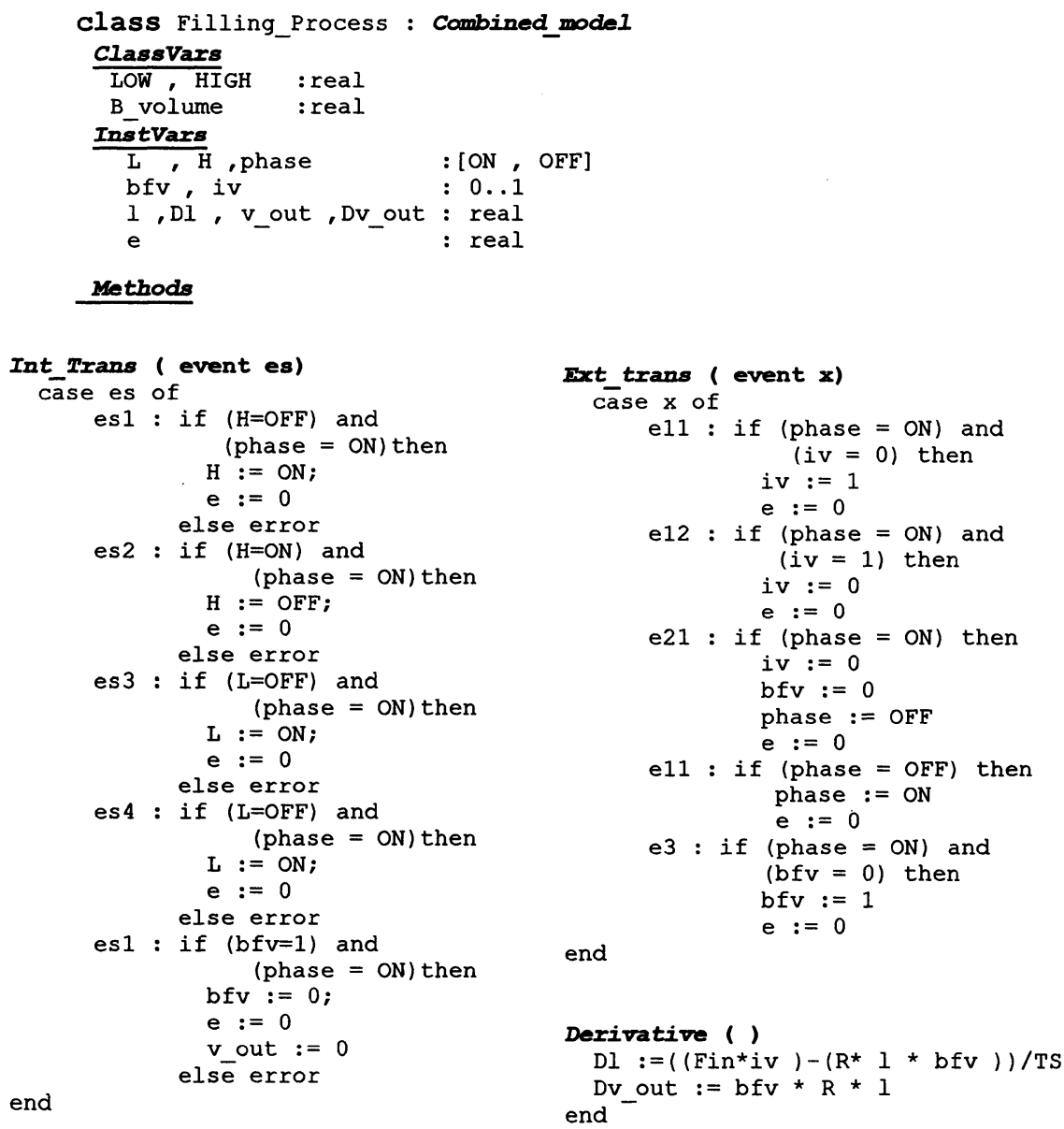




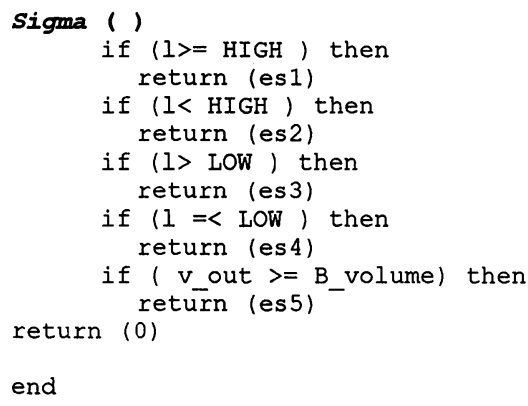

The specialization gives the specific definitions by overriding the virtual methods Int_Trans, Ext_Trans and Output_Func, of the class atomic and Derivative and Sigma of the class Combined model

\subsection{Abstract simulator principles}

The abstract simulators are defined as the interpretation of dynamic behaviours specified by models. Essentially, they are objects whose methods are an algorithmic description of how to carry out the instructions implicit in models to generate their behavior. Simulators are assigned to handle basic models in a one-to-one manner. The whole simulation program is then represented by a network of such (model, simulator) pairs.

There are two types of abstract simulator: Simulators and Coordinators; assigned to handle atomic-DEVS models, and coupled models in a one-to-one manner respectively. Simulation proceeds by messages that carry data and synchronization information passed among the abstract simulators.

\subsubsection{The. Abstract Simulator of Discrete Event System}

The operation of an abstract simulator involves four types of messages : *, $\mathrm{x}, \mathrm{y}$ and donemessages. It consists of two methods that handle four main variables :

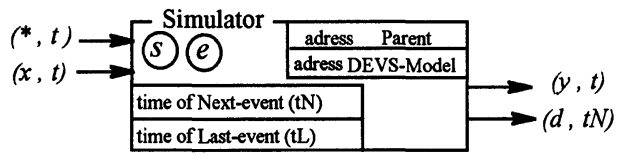

Figure 5. Discrete Event Simulator

$-s:$ is the sequential state variable,

- e : the elapsed time since the last state transition, 
$-\mathrm{tL}$ : the time of the last event and

$-\mathrm{tN}$ : is the time of the next internal state transition.

The algorithm of the abstract simulator is divided onto two parts : One method is activated on a reception of an $(x, t)$ message, and the other one corresponds to an $(*, t)$ message.

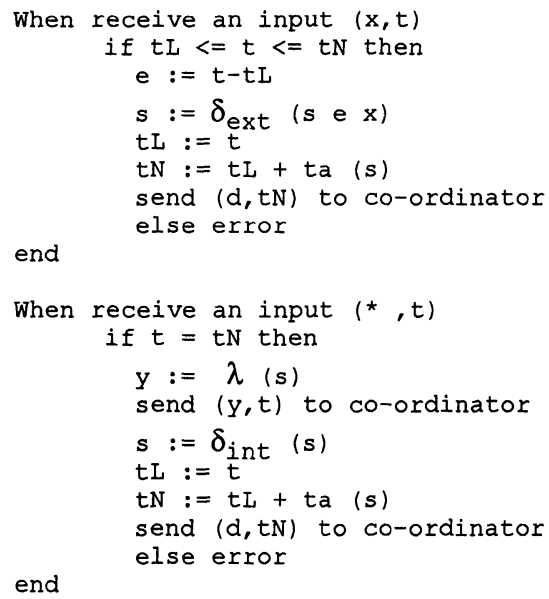

In (Zeigler, 1984), the abstract simulator was shown to satisfy the criterion of correct simulation of a DEVS model. The *-message indicates that an internal event shall be executed and is transmitted to the imminent component. If there are more than one imminent component, the tie breaking function Select is used to select one of them. When a *-message is received by a simulator, it sends its output as a y-message and carries out the internal transition in the associated DEVS model. The output (y-message) is sent back to the parent coordinator which consults the external output coupling and the internal couplings to obtain the addresses to which the message should be sent as an $x$-message. When a Coordinator receives an $x$-message, it consults the external input coupling to generate the appropriate $x$-message for the subordinate influenced by the external event. When a Simulator receives an $\mathrm{x}$-message, it executes the external transition of the associated DEVS. A done-message indicates the completion of the state transition and contains the time of the next internal transition.

\subsubsection{The Abstract Simulator for Combined Model}

The simulators described in the preceding paragraph, are defined to handle discrete event systems. So to simulate a combined discrete event-continuous state system, we must define a special simulator which has methods to compute the continuous state values during the observation time.

The extension retains the basic structure of the abstract simlators. Since the state-events are not time scheduled events, a simulator for a combined model can not determine the time of the next internal transition. To solve this problem, we use a sampling method which predicts the occurrence of an internal event with an estimated time $t N e$. If a state event occurs at time $t<t N e$, a time warp mechanism is used to correctly simulate a multicomponent model. The mechanism to rollback an object is the heart of the time warp. The time warp exerts no effort 
to ensure that messages are delivered to an object in increasing timestamp order. Instead, the simulation proceeds on the assumption that there will be no stragglers (messages with time stamps less than the local time ). But if a straggler should arrive at time $t$, the object must roll back to the time $t$ and cancel all the side effects that occurred as a result of processing messages with timestamps greater than $t$ (Jefferson, $85,87,89$ ).

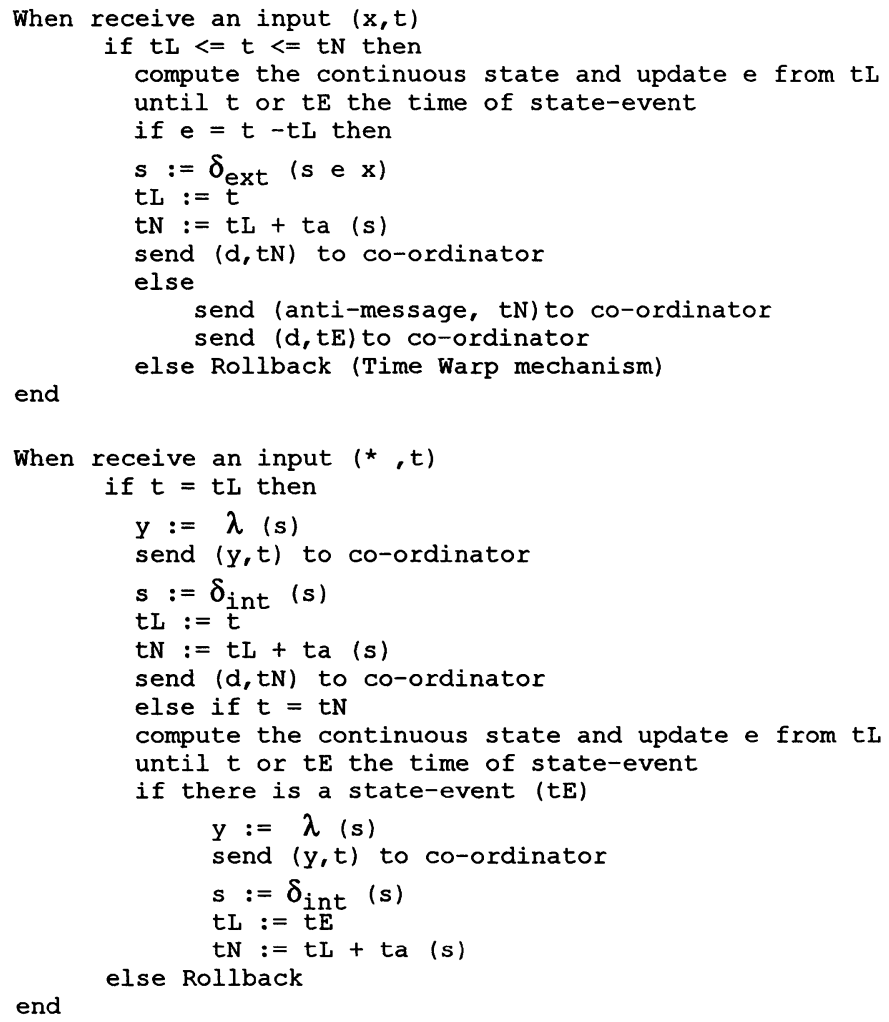

end

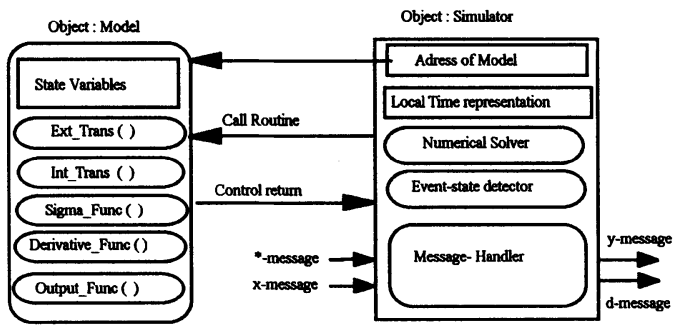

Figure 6. A model -Simulator pair. 
Note that:

- The messages exchanged among the abstract simulators are timestamped.

- When a simulator of combined model receives an $(x, t)$ or $\left({ }^{*}, t N e\right)$ message, before executing the external or internal transition it updates its local clock by employing the solver based on a numerical integration method to compute the continuous states value step by step and the event detector method to check the value of the sigma function. If a state event occurs at time $t N<t N e$, the simulator transmits an $\left({ }^{*}, t N\right)$ to the parent coordinator (figure 7).

- When a Coordinator receives a message with a timestamp $t N$ less than its local clock, it releases the rollback mechanism by means of the antimessages, and start again with the new value $t N$ of the internal state-event.

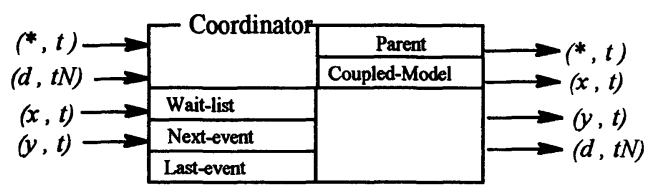

Figure 7. The Coordinator of Coupled Model.

\section{CONCLUSION}

A variety of paradigms are being actively explored to deal with systems where a large number of discrete events and continuous dynamic behaviours are simultaneously important. An extension of the DEVS formalism shows how it is possible to express models of combined discrete event-continuous systems, so that the whole formalism guarantees the strength of the theoretical based methods for simulation. By the bottle-filling process example, we have illustrated that the proposed modeling framework makes a clear description of complex combined systems possible. The object oriented paradigm provides convenient tools to carry out this abstract formalism concept and improves the isomorphic relation between the model and its representation in the simulation program.

\section{REFERENCES}

Alla H. (1994) Les Réseaux de Petri: des Evolutions Particulièrement Adaptées aux Systèmes Hybrides. in ADPM94, Les Systèmes Dynamiques Hybrides.

Buisson J. (1993) Analysis and Characterisation of Hybrid Systems with Bond-Graphs. in IEEE International Conf. on Systems, Man and Cybernetics, Vol 1.

Caristi J. and Sands D.C. (1991) Simulation of Epiphytic Bacterial Growth under Field Conditions Simulation J..

Cellier F.E. (1979) Combined Continuous/Discrete System Simulation : Languages, Usefulness, Experiences and Future Development in Methodology in Systems Modelling and Simulation, Eds. B.P. Zeigler, M.S. Elzas, G.J. Klir \& T.I. Ören, North Holland Pub.,. 
Concepcion A.I.(1985) The Implementation of the Hierarchical Abstract Simulator on The HEP Computer. Proc. Winter Simulation Conf.

Concepcion A.I. and Zeigler B. P.(1988) DEVS Formalism: A Framwork for Hierarchical Model Devlopement: IEEE Transaction on Software Engineering, Vol. 14, NO. 2.

Fahrland D. (1970) Combined Discrete Event Continuous Systems Simulation. Simulation J..

Jefferson D. R. and Sowizral H. (1985a) Fast Concurrent Simulation using the Time Warp Mechanism. In Proc. SCS conf. on Distributed Simulation, San Diego.

Jefferson D. R. (1985b) Virtuel Time. ACM Transaction on Programming Languages and Systems, Vol. 7, No. 3.

Jefferson D. R., Backman B.and al (1987) The Time Warp Operating System. 11th Symp. Operating Systems Principles, Vol 21.

Jefferson D. R. and al (1989) The Performance of a Distributed Combat Simulation with the Time Warp Operating System. Concurrency Pratice and Experience, Vol. 1, No. 1.

Kim T.G. (1988) Knowledge-based environment for hierarchical modelling and simulation. $\mathrm{Ph}$. D. dissertation, University of Arizona, Tucson, AZ.

Kim T.G.and Zeigler B.P. (1989) ESP-Scheme : A Realization of System Entity Structure in a LISP Environment. Proc. AI and Simulation Multiconference, SCS Publications, San Diego.

Praehofer H. (1990) System Theoretic Formalisms for Combined Discrete-Continuous System Simulation. Int. J. General Systems, Vol 19, 219-240.

Praehofer H. (1992) An Environment for DEVS-Based Multiformalism Simulation in Common Lisp. Special issue on : Software Environment for Discrete-Event Dynamic Systems.

Stiver J.A. and Antsaklis P.J. (1993) On the Controllability of Hybrid Control Systems. in 32nd IEEE Conf. on Decision and Control.

Teggar M. (1996) Modélisation et Simulation des Systèmes Dynamiques Hybride. Thèse de Doctorat de l'université de Valenciennes et du Hainaut Combresis.

Wang Q. (1989) Management of Continuous Models in DEVS-Scheme: Time Windows for Event-Based Control Masters Thesis, Dept. of Electrical an Computer Engineering, University of Arizona, Tucson, AZ.

Zeigler B.P. (1976) Theory of Modelling and Simulation. J. Wiley NY . (Reissued by Krieger Pub. Co., Malabar, FL, 1985).

Zeigler B.P. (1984) Multifacetted Modelling and Discrete Event Simulation. Academic Press, London.

Zeigler B.P. (1987) Hierarchical, Modular Discrete Event Simulation in an Object-Oriented Environement. Simulation Journal, Vol. 49:5.

Zeigler B.P. (1989) DEVS Representation of Dynamical systems : Event-Based Intelligent Control. Proceedings Of The IEEE, Vol. 77, NO. 1.

Zeigler B.P. (1990) Object-Oriented Simulation with Hierarchical, Modular Models. Academic Press, London. 